\title{
A Study on Gender and Vision Differences in Terms of the Center of Gravity Sway in Adults' Standing
}

\author{
Yoshinori Nagasawa $^{1, *}$, Shinichi Demura ${ }^{2}$, Hiroshi Hirai $^{3}$ \\ ${ }^{1}$ Department of Health and Sports Sciences, Kyoto Pharmaceutical University, Kyoto, Kyoto, Japan. \\ ${ }^{2}$ College of Human and Social Sciences, Kanazawa University, Kanazawa, Ishikawa, Japan. \\ ${ }^{3}$ Higher Education Development, Osaka Prefecture University, Sakai, Osaka, Japan.
}

How to cite this paper: Yoshinori Nagasawa, Shinichi Demura, Hiroshi Hirai. (2021). A Study on Gender and Vision Differences in Terms of the Center of Gravity Sway in Adults' Standing. The Educational Review, USA, 5(12), 451-459. DOI: 10.26855/er.2021.12.001

Received: November 11, 2021

Accepted: December 2, 2021

Published: December 10, 2021

Corresponding author: Yoshinori Nagasawa, Department of Health and Sports Sciences, Kyoto Pharmaceutical University, Kyoto, Kyoto, Japan.

Email: ynaga@mb.kyoto-phu.ac.jp

\section{Abstract}

In a standing posture, the human center of gravity shaking (CGS) changes constantly; its variation increases depending on the disturbance stimulus. When eyes are closed, the standing posture becomes unstable because of restricted information feedback from visual cues. In this investigation, we assumed that gender differences in the CGS variables and their relationships differed when the eyes were open and when they were closed. We examined the gender differences in the CGS variables and the relationships among them when the subjects' eyes were open and then closed while standing. Do the gender and vision differences exists in terms of gravity sway with 81 healthy participants aged 21-32 years when their eyes were open and shut? The participants maintained a Romberg posture (standing posture with feet closed) for $1 \mathrm{~min}$ on the measuring equipment in two different conditions: with eyes opened as well as closed. The $\mathrm{x}$-axis, y-axis, total trajectory lengths, and outer peripheral area (general sway variable: GS variable) were input to a computer with a sampling rate of $20 \mathrm{~Hz}$. A peak power frequency (peak) and peak power spectrum value (power) appeared in three directions (X, Y, and R) and were computed as power spectrum variables (PS variable). The measurement order was randomized in both conditions. We performed three trials for both conditions with a $1 \mathrm{~min}$ rest between each trial. The mean of the last two trials became the evaluation variables. A two-way analysis of variance and multiple comparison tests resulted in the following: 1) the y-axis trajectory length had a higher value for men with eyes closed and in both genders with eyes closed; 2) the total trajectory length had a higher value for men under both conditions and in both genders with eyes closed; 3) the outer peripheral area had a higher value only for men with closed eyes; 4) the $\mathrm{x}$-axis trajectory length and $\mathrm{x}$-direction power had higher values for men and those with closed eyes; 5) the peak and power of $x$-direction and the peak of r-direction also had higher values for eyes closed than for open conditions; and 6) the power of $x-, y-$, and r-directions had higher values in men than in women. The results from the correlation analysis showed that the GS variables exhibited mutually significant correlations during and between both conditions in both genders. The PS variables showed significant correlations between y- and r-direction powers in both genders with eyes open and closed, only in the x-direction power between eyes open and closed in men, and in only the y-direction peak between eyes 
open and closed in women. Most CGS variables show a larger variation when eyes are closed than when eyes are open; further the variations occur more so for men than for women. Relationships between CGS variables with both conditions were visible in both genders, just that the relationships differed between the GS and PS variables.

\section{Keywords}

Humans, Functional Balance, Stability, Eyes-Open-and-Closed, Analysis of Variance

\section{Introduction}

The center of gravity shaking (CGS) while standing is determined by the posture of the body and skeletal muscle alignment according to Pascoe et al. (1997). It depends on reflex control based on afferent inputs from the visual, vestibular (semi-circular duct), and somatosensory systems (Vuillerme \& Nougier, 2003; Fransson et al., 2004). Fatigue, drinking, and aging are other factors that impact CGS (Kitabayashi et al., 2004; Noda et al., 2004; Demura et al., 2008; Degache et al., 2020).

Visual cues contribute significantly to posture control (Siriphorn et al., 2015). Therefore, the center of gravity sways constantly to maintain a stable standing posture. If feedback information is lacking because of constraints in visual senses or field, the center of gravity position tends to fluctuate. Closed eyes have a notable impact on restricting visual information and a significant effect on the variation of center of gravity (Grace Gaerlan et al., 2012).

Human's CGS primarily uses the center of pressure (Demura et al., 2006; Masani et al., 2007) and has been evaluated using general sway (GS) variables [such as before/after, left/right, total trajectory lengths, moving velocity, and area component; (Hill \& Price, 2018)]. Uchiyama and Demura (2007) reported that a variation of the before/after and left/right directions increased in subjects with eyes closed owing to the blocked visual senses, as compared to those with eyes open. Chen et al. (2012) reported that the elderly with subpar visual senses and fields have a larger CGS, such as distance and area, during a standing posture than those with undiminished visual capacity. On the basis of these findings, we conclude that CGS is affected by visual information, and the affected CGS variables differ when the eyes are open or shut.

CGS variables are compelling in quantitative estimations of shaking distance and scope. However, it is difficult to differentiate whether the velocity is high and the shaking is wiggling in before/after and left/right directions (high-frequency shaking) or the velocity is low and the shaking is increased (low-frequency shaking). Periodic CGS was visible in patients with dizziness and equilibrium disorder (Taguchi et al., 1986) and the PS variable identified dizziness and equilibrium disorder (Demura et al., 2008). In healthy people, the spectrum density concentrates on the low-frequency passband. It becomes high in the middle- and high-frequency passband in patients with Meniere's disease (Yoneda \& Tokumasu, 1986). Even in healthy people, the spectrum density may appear in the middle- and high-frequency passband when eyes are closed and the visual sense blocked as those in people with dizziness and equilibrium disorder.

Kitabayashi et al. (2004) reported gender differences in the CGS. However, Demura et al. (2008) recorded no significant gender differences in the CGS PS. CGS variables with gender differences differ when eyes are open or shut. However, the gender difference of the CGS variables when eyes are closed has not been thoroughly examined.

We hypothesized that the CGS variables (GS and PS variables) are higher when eyes were closed than when they were open (hypothesis 1) and more in men than in women (hypothesis 2). The CGS variables have mutually high relationships when eyes are open and closed, but the relationships among GS and PS variables are low (hypothesis 3). The relationship between CGS variables with eyes open and closed is also minimal (hypothesis 4).

This study examined gender differences in CGS variables when eyes were open and shut in a standing posture and the relationships among CGS variables amongst young adult men and women.

\section{Methods}

\subsection{Participants}

The participants were 81 healthy university students (age 21-32, mean age $=23.3$ years, standard deviation [SD] $=$ 2.3 years): 43 men (mean age $=24$ years, $S D=2.9$ years) and 38 women (mean age $=22.5$ years, $S D=0.9$ years) (Table 1). 
Table 1. Physical characteristics of participants

\begin{tabular}{ccccccccc}
\hline & \multicolumn{2}{c}{ All $(\mathrm{n}=81)$} & \multicolumn{2}{c}{ Men $(\mathrm{n}=43)$} & \multicolumn{2}{c}{ Women $(\mathrm{n}=38)$} & \multicolumn{2}{c}{ Gender difference } \\
& $M$ & $S D$ & $M$ & $S D$ & $M$ & $S D$ & $t$ & $E S$ \\
\hline Age (years) & 23.3 & 2.30 & 24.0 & 2.89 & 22.5 & 0.89 & $3.24^{*}$ & 2.19 \\
Height $(\mathrm{cm})$ & 164.6 & 7.92 & 169.4 & 6.79 & 159.2 & 5.20 & $7.49^{*}$ & 6.10 \\
Body weight $(\mathrm{kg})$ & 56.8 & 7.09 & 58.6 & 5.67 & 54.7 & 7.99 & $2.55^{*}$ & 6.86 \\
\hline
\end{tabular}

M: mean, SD: standard deviation, ES: effect size, ${ }^{*}$ : $p<0.05$

The mean values for height and body mass were similar to Japanese normative values of the same age (Society for Physical Fitness Standards Research in Tokyo Metropolitan University, 2000). None of the participants reported previous wrist injuries or upper limb nerve damage, and all were in good health. The purpose and study procedure were explained in detail to all participants in advance, and all provided informed and signed consent. The experimental protocol was approved by the Ethical Review Committee for Medical and Health Research Involving Human Subjects of Japanese Society of Test and Measurement in Health and Physical Education (approval number: 2018-001) and Kyoto Pharmaceutical University (approval number: 18-17-11). None of the participants had previously undergone a CGS test.

\subsection{The measuring apparatus for the CGS}

A measuring instrument measured the center of gravity trajectory with a laptop. It calculated the participants' center of gravity while standing on a measuring plate; the position was projected on the plantar surface by four vertical load sensors built on a rectangular plate (the $360 \mathrm{~mm}$ square) on a horizontal plane. The center of gravity trajectory values were transmitted to a computer at a sampling rate of $20 \mathrm{~Hz}$ through a USB data output cable after A/D conversion. The CGS apparatus was previously described in detail (Demura et al., 2006; Noguchi et al., 2013).

\subsection{Testing and measuring the CGS}

The participants maintained a Romberg posture (a standing posture with feet closed) with eyes open for 1 min on the CGS apparatus (TKK-5810, Takei-kiki industry corporation) in eyes open and closed conditions. The measurement order was randomized in both conditions. We recorded the measurement once we confirmed that the participant was holding a standing posture based on the point of gaze set at eye level. Three measurements were taken with a 1-min rest between the trials for both conditions.

\subsection{Estimation variables}

The CGS during the eyes open condition was estimated using the trajectory lengths of before/after and left/right direction, total trajectory lengths, moving speed, and area component (GS variable) displaying the distance and scope of the center of pressure shaking (Hill \& Price, 2018). Since healthy people have a high speed and twitch shaking in before/after and left/right direction (high-frequency shaking), but the patients with dizziness and equilibrium disorder have a low speed and large shaking (low-frequency shaking), the PS variables were used to estimate the periodic CGS in the patients (Demura et al., 2008). We hypothesized that the same frequency CGS variation was present in healthy people during eyes closed trials. Therefore, the GS variables (x-axis, y-axis, total trajectory lengths, and outer peripheral area) and the PS variables became estimation variables. They were transmitted to a computer at a sampling rate of $20 \mathrm{~Hz}$. An average of the last two trials in each condition (eyes open and closed) was used for analysis. Within the GS variables, each trajectory length indicated the distance of shaking. The outer peripheral area signified the shaking space of the combined x-axis (left and right) and y-axis (backward and forward) directions. We conducted a power spectral analysis by conditions of no window function and 1024 data. A peak power frequency (peak: $\mathrm{Hz}$ ) and peak power spectrum value (power: $\mathrm{mm}$ ) appeared in three directions (X, Y, and R) and computed as the PS variables. In addition, based on the division of Kapteyn et al. (1983) used in many previous studies, the percentage of total power with powers of three frequency $(0.02-0.2,0.2-2$, and 2-10 Hz) spectra were computed.

\subsection{Statistical analysis}

Data was analyzed using SPSS version 23.0 for Windows software (International Business Machines Corporation). Descriptive statistics were reported as mean \pm SD. A two-way analysis of variance (ANOVA) examined significant differences between the genders and eye conditions for each estimate variable. To derive telling interaction and main effects, we used a multiple comparison test using the Bonferroni method for pairwise comparisons. Pearson correlations 
examined the relationships among variable estimations in both conditions by gender; $\mathrm{p}<0.05$ provided statistical significance.

\section{Results}

\subsection{Gender and vision condition differences (eyes open and closed) of CGS variables}

Table 2 shows the means and SDs for each CGS (GS and PS) variable by gender and vision factors. Table 3 shows the results of the two-way ANOVA for each CGS variable.

In the results of the ANOVA, significant interactions were found for the y-axis, total trajectory lengths, and outer peripheral area. Telling main effects were found for the $\mathrm{x}$-axis trajectory length and $\mathrm{x}$-direction power for the gender and vision factors. Multiple comparison tests showed that the y-axis trajectory length had a higher value for men with closed eyes and in both genders with closed eyes. The total trajectory length had a higher value for men under both conditions and in both genders with closed eyes. The outer peripheral area had a higher value only for men with closed eyes. The $\mathrm{x}$-axis trajectory length and $\mathrm{x}$-direction power had higher values for men and for those with their eyes closed.

Significant main effects were found for the peak and power of $\mathrm{x}$-direction and the peak of r-direction for the vision factor and seen for the power of $\mathrm{x}-, \mathrm{y}$-, and r-directions for the gender factor. Multiple comparison tests showed that the peak and power of $\mathrm{x}$-direction and the peak of r-direction had higher values for eyes closed than open conditions. The power of $\mathrm{x}-$, $\mathrm{y}-$, and $\mathrm{r}$-directions had higher values in men than in women.

Table 2. Means and standard deviations in CGS variables during eyes open and closed in all participants and by gender

\begin{tabular}{|c|c|c|c|c|c|c|c|c|c|c|c|c|}
\hline & \multicolumn{2}{|c|}{ All $(\mathrm{n}=81)$} & \multicolumn{2}{|c|}{ Men $(n=43)$} & \multicolumn{2}{|c|}{ Women $(n=38)$} & \multicolumn{2}{|c|}{ All $(\mathrm{n}=81)$} & \multicolumn{2}{|c|}{ Men $(n=43)$} & \multicolumn{2}{|c|}{ Women $(\mathrm{n}=38)$} \\
\hline & $M$ & $S D$ & $M$ & $S D$ & $M$ & $S D$ & $M$ & $S D$ & $M$ & $S D$ & $M$ & $S D$ \\
\hline & \multicolumn{2}{|c|}{ Eyes open } & \multicolumn{10}{|c|}{ Eyes closed } \\
\hline $\mathrm{X}$-axis trajectory length [mm] & 258.2 & 68.82 & 276.7 & 70.87 & 237.4 & 60.82 & 351.9 & 110.29 & 384.2 & 120.07 & 315.4 & 85.80 \\
\hline Y-axis trajectory length [mm] & 202.3 & 51.11 & 211.8 & 55.29 & 191.7 & 44.25 & 292.2 & 85.40 & 322.7 & 97.29 & 257.6 & 52.12 \\
\hline Total trajectory length [mm] & 363.7 & 91.21 & 386.1 & 96.37 & 338.4 & 78.80 & 507.6 & 146.09 & 556.1 & 162.27 & 452.7 & 102.09 \\
\hline Outer peripheral area $\left[\mathrm{mm}^{2}\right]$ & 252.7 & 144.94 & 285.4 & 167.49 & 215.8 & 104.56 & 413.7 & 321.10 & 516.6 & 395.26 & 297.3 & 139.17 \\
\hline \multicolumn{13}{|l|}{$\mathrm{X}$-direction } \\
\hline Peak [Hz] & 0.04 & 0.03 & 0.04 & 0.04 & 0.04 & 0.03 & 0.05 & 0.04 & 0.06 & 0.05 & 0.05 & 0.04 \\
\hline Power [mm] & 2.77 & 1.48 & 3.08 & 1.80 & 2.42 & 0.91 & 3.12 & 1.61 & 3.50 & 1.75 & 2.69 & 1.34 \\
\hline $\begin{array}{l}\text { Percentage of total power with powers of } \\
\qquad 0.02-0.2 \mathrm{~Hz}[\%]\end{array}$ & 21.86 & 4.87 & 21.76 & 5.12 & 21.97 & 4.62 & 22.11 & 5.89 & 22.67 & 6.60 & 21.47 & 4.97 \\
\hline $\begin{array}{l}\text { Percentage of total power with powers of } \\
\qquad 0.2-2 \mathrm{~Hz}[\%]\end{array}$ & 57.84 & 4.22 & 58.39 & 3.97 & 57.21 & 4.45 & 58.42 & 4.73 & 57.48 & 4.89 & 59.49 & 4.36 \\
\hline $\begin{array}{l}\text { Percentage of total power with powers of } \\
\qquad 2-10 \mathrm{~Hz}[\%]\end{array}$ & 20.31 & 2.94 & 19.85 & 2.72 & 20.82 & 3.12 & 19.47 & 3.98 & 19.85 & 4.88 & 19.04 & 2.62 \\
\hline \multicolumn{13}{|l|}{ Y-direction } \\
\hline Peak [Hz] & 0.04 & 0.03 & 0.03 & 0.01 & 0.04 & 0.03 & 0.03 & 0.02 & 0.03 & 0.02 & 0.04 & 0.03 \\
\hline Power [mm] & 3.99 & 1.74 & 4.37 & 1.96 & 3.56 & 1.35 & 4.09 & 1.56 & 4.42 & 1.58 & 3.73 & 1.48 \\
\hline $\begin{array}{l}\text { Percentage of total power with powers of } \\
\qquad 0.02-0.2 \mathrm{~Hz}[\%]\end{array}$ & 30.74 & 5.94 & 30.16 & 6.54 & 31.40 & 5.18 & 25.83 & 5.56 & 25.60 & 6.71 & 26.08 & 3.96 \\
\hline $\begin{array}{l}\text { Percentage of total power with powers of } \\
\qquad 0.2-2 \mathrm{~Hz}[\%]\end{array}$ & 48.27 & 4.62 & 48.34 & 4.95 & 48.18 & 4.30 & 54.44 & 4.40 & 53.84 & 4.79 & 55.11 & 3.87 \\
\hline $\begin{array}{l}\text { Percentage of total power with powers of } \\
\qquad 2-10 \mathrm{~Hz}[\%]\end{array}$ & 20.99 & 4.00 & 21.49 & 4.41 & 20.42 & 3.44 & 19.74 & 5.09 & 20.56 & 6.34 & 18.81 & 2.93 \\
\hline \multicolumn{13}{|l|}{ R-direction } \\
\hline Peak [Hz] & 0.04 & 0.02 & 0.03 & 0.01 & 0.04 & 0.03 & 0.05 & 0.03 & 0.04 & 0.03 & 0.05 & 0.04 \\
\hline Power [mm] & 3.28 & 1.33 & 3.43 & 1.43 & 3.10 & 1.21 & 3.11 & 1.28 & 3.43 & 1.35 & 2.76 & 1.10 \\
\hline $\begin{array}{l}\text { Percentage of total power with powers of } \\
\qquad 0.02-0.2 \mathrm{~Hz}[\%]\end{array}$ & 27.19 & 5.47 & 26.42 & 5.84 & 28.06 & 4.94 & 23.00 & 5.38 & 22.78 & 5.91 & 23.25 & 4.79 \\
\hline $\begin{array}{l}\text { Percentage of total power with powers of } \\
\qquad 0.2-2 \mathrm{~Hz}[\%]\end{array}$ & 51.65 & 4.26 & 52.11 & 4.49 & 51.13 & 3.97 & 56.35 & 4.19 & 56.00 & 4.41 & 56.75 & 3.95 \\
\hline $\begin{array}{l}\text { Percentage of total power with powers of } \\
\qquad 2-10 \mathrm{~Hz}[\%]\end{array}$ & 21.16 & 3.85 & 21.47 & 4.33 & 20.81 & 3.25 & 20.65 & 4.78 & 21.23 & 5.98 & 20.00 & 2.82 \\
\hline
\end{tabular}


Table 3. Results of two-way analysis of variance

\begin{tabular}{|c|c|c|c|c|c|c|c|}
\hline Variable & Factor & $\mathrm{df}$ & $\mathrm{F}$ & $\mathrm{p}$ & & Partial $\eta^{2}$ & Multiple comparisons \\
\hline \multirow[t]{5}{*}{$\mathrm{X}$-axis trajectory length } & Sex & 1 & 8.949 & 0.004 & $*$ & 0.102 & Men $>$ Women \\
\hline & Error & 79 & $(6572.47)$ & & & & \\
\hline & Vision & 1 & 146.485 & 0.000 & $*$ & 0.650 & Eyes open $<$ Eyes closed \\
\hline & Sex $\times$ vision & 1 & 3.695 & 0.058 & & 0.045 & \\
\hline & Error & 79 & $(8756.34)$ & & & & \\
\hline \multirow[t]{5}{*}{ Y-axis trajectory length } & Sex & 1 & 10.123 & 0.002 & $*$ & 0.114 & Eyes closed: Men > Women \\
\hline & Error & 79 & (7221.21) & & & & \\
\hline & Vision & 1 & 194.105 & 0.000 & $*$ & 0.711 & Men: Eyes open < Eyes closed \\
\hline & Sex $\times$ vision & 1 & 12.566 & 0.001 & $*$ & 0.137 & Women: Eyes open < Eyes closed \\
\hline & Error & 79 & (1625.96) & & & & \\
\hline \multirow[t]{5}{*}{ Total trajectory length } & Sex & 1 & 10.221 & 0.002 & $*$ & 0.115 & Eyes open: Men > Women \\
\hline & Error & 79 & $(22530.48)$ & & & & Eyes closed: Men > Women \\
\hline & Vision & 1 & 194.289 & 0.000 & $*$ & 0.711 & Men: Eyes open < Eyes closed \\
\hline & Sex $\times$ vision & 1 & 7.481 & 0.008 & $*$ & 0.087 & Women: Eyes open < Eyes closed \\
\hline & Error & 79 & (4194.11) & & & & \\
\hline \multirow[t]{5}{*}{ Outer peripheral area } & Sex & 1 & 9.441 & 0.003 & $*$ & 0.107 & Eyes closed: Men > Women \\
\hline & Error & 79 & (89173.68) & & & & \\
\hline & Vision & 1 & 42.913 & 0.000 & $*$ & 0.352 & Men: Eyes open < Eyes closed \\
\hline & Sex $\times$ vision & 1 & 9.822 & 0.002 & $*$ & 0.111 & \\
\hline & Error & 79 & (22991.39) & & & & \\
\hline \multirow[t]{5}{*}{ Peak of x-direction } & Sex & 1 & 0.051 & 0.822 & & 0.001 & \\
\hline & Error & 79 & $(0.00155)$ & & & & \\
\hline & Vision & 1 & 4.090 & 0.047 & $*$ & 0.049 & Eyes open $<$ Eyes closed \\
\hline & Sex $\times$ vision & 1 & 0.541 & 0.464 & & 0.008 & \\
\hline & Error & 79 & $(0.00184)$ & & & & \\
\hline \multirow[t]{5}{*}{ Power of x-direction } & Sex & 1 & 5.857 & 0.018 & $*$ & 0.069 & Men > Women \\
\hline & Error & 79 & (3.70443) & & & & \\
\hline & Vision & 1 & 5.659 & 0.020 & $*$ & 0.067 & Eyes open $<$ Eyes closed \\
\hline & Sex $\times$ vision & 1 & 0.273 & 0.603 & & 0.003 & \\
\hline & Error & 79 & $(0.86820)$ & & & & \\
\hline \multirow[t]{5}{*}{ Peak of y-direction } & Sex & 1 & 2.850 & 0.095 & & 0.035 & \\
\hline & Error & 79 & $(0.00086)$ & & & & \\
\hline & Vision & 1 & 1.593 & 0.211 & & 0.019 & \\
\hline & Sex $\times$ vision & 1 & 0.901 & 0.345 & & 0.011 & \\
\hline & Error & 79 & $(0.00036)$ & & & & \\
\hline \multirow[t]{5}{*}{ Power of y-direction } & Sex & 1 & 7.129 & 0.009 & $*$ & 0.083 & Men > Women \\
\hline & Error & 79 & (3.16271) & & & & \\
\hline & Vision & 1 & 0.216 & 0.643 & & 0.003 & \\
\hline & Sex $\times$ vision & 1 & 0.076 & 0.783 & & 0.001 & \\
\hline & Error & 79 & (2.08323) & & & & \\
\hline \multirow[t]{5}{*}{ Peak of r-direction } & Sex & 1 & 2.403 & 0.125 & & 0.029 & \\
\hline & Error & 79 & $(0.00109)$ & & & & \\
\hline & Vision & 1 & 4.494 & 0.037 & $*$ & 0.053 & Eyes open $<$ Eyes closed \\
\hline & Sex $\times$ vision & 1 & 0.010 & 0.919 & & 0.000 & \\
\hline & Error & 79 & $(0.00073)$ & & & & \\
\hline \multirow[t]{5}{*}{ Power of r-direction } & Sex & 1 & 5.072 & 0.027 & $*$ & 0.060 & Men > Women \\
\hline & Error & 79 & $(1.97915)$ & & & & \\
\hline & Vision & 1 & 0.910 & 0.343 & & 0.011 & \\
\hline & Sex $\times$ vision & 1 & 0.910 & 0.343 & & 0.011 & \\
\hline & Error & 79 & (1.33718) & & & & \\
\hline
\end{tabular}

$*: \mathrm{p}<0.05$ 


\subsection{Relationships among the CGS variables}

Table 4 shows Pearson's correlation coefficients among CGS variables by gender. In men, significant correlations ( $\mathrm{r}$ $=0.63-0.98$ ) over a moderate degree for GS variables were found among GS variables in eyes open and closed conditions, and significant correlations $(\mathrm{r}=0.48-0.80)$ over a moderate degree were found between GS variables of both conditions. Significant correlations ( $r=0.76$ and $r=0.77$ ) over a moderate degree for PS variables were found only between the power of $y$ - and r-directions in both conditions and significant correlation was found only in the power of $\mathrm{x}$-direction between both conditions $(\mathrm{r}=0.71)$. The GS variables showed significant and moderate correlations (eyes open: $r=0.42-0.74$, eyes closed: $r=0.42-0.74$ ), with the power of $x$-direction in both conditions, but not in the peak of $\mathrm{x}-\mathrm{r}-$, and $\mathrm{y}$-directions.

Table 4. Pearson's correlation coefficients in each CGS variables by gender

\begin{tabular}{|c|c|c|c|c|c|c|c|c|c|c|c|c|c|c|c|c|c|c|c|c|c|c|c|c|}
\hline & & & 1 & & 2 & & 3 & & 4 & & 5 & & 6 & & 7 & & 8 & & 9 & & 10 & & 11 & \\
\hline 1 & Eyes open & $\mathrm{X}$-axis trajectory length & 1.00 & & 0.80 & $*$ & 0.97 & $*$ & 0.61 & $*$ & & & & & & & & & & & 0.39 & $*$ & 0.88 & $*$ \\
\hline 2 & & Y-axis trajectory length & 0.88 & $*$ & 1.00 & & 0.93 & $*$ & 0.72 & $*$ & & & & & & & 0.42 & $*$ & & & 0.47 & $*$ & 0.70 & $*$ \\
\hline 3 & & Total trajectory length & 0.98 & $*$ & 0.96 & $*$ & 1.00 & & 0.69 & $*$ & & & & & & & 0.36 & $*$ & & & 0.44 & $*$ & 0.85 & $*$ \\
\hline 4 & & Outer peripheral area & 0.63 & $*$ & 0.77 & $*$ & 0.71 & $*$ & 1.00 & & & & 0.58 & $*$ & & & 0.77 & $*$ & & & 0.76 & $*$ & 0.45 & $*$ \\
\hline 5 & & Peak of $\mathrm{x}$-direction & & & & & & & & & 1.00 & & -0.40 & $*$ & 0.59 & $*$ & & & 0.61 & $*$ & & & & \\
\hline 6 & & Power of $x$-direction & 0.42 & $*$ & 0.53 & $*$ & 0.47 & $*$ & 0.74 & $*$ & -0.34 & $*$ & 1.00 & & & & 0.33 & $*$ & & & 0.34 & $*$ & & \\
\hline 7 & & Peak of y-direction & & & & & & & & & & & & & 1.00 & & & & 0.90 & $*$ & & & & \\
\hline 8 & & Power of y-direction & & & & & & & 0.49 & $*$ & & & & & -0.47 & $*$ & 1.00 & & & & 0.89 & * & & \\
\hline 9 & & Peak of r-direction & & & & & & & & & & & & & 0.65 & $*$ & -0.35 & $*$ & 1.00 & & & & & \\
\hline 10 & & Power of r-direction & & & & & & & 0.36 & $*$ & & & & & -0.37 & $*$ & 0.76 & $*$ & -0.49 & $*$ & 1.00 & & & \\
\hline 11 & Eyes closed & $\mathrm{X}$-axis trajectory length & 0.72 & $*$ & 0.69 & $*$ & 0.73 & $*$ & 0.52 & $*$ & & & 0.34 & $*$ & & & & & & & & & 1.00 & \\
\hline 12 & & Y-axis trajectory length & 0.48 & $*$ & 0.66 & $*$ & 0.58 & $*$ & 0.49 & $*$ & & & & & & & & & & & & & 0.80 & $*$ \\
\hline 13 & & Total trajectory length & 0.65 & $*$ & 0.72 & $*$ & 0.70 & $*$ & 0.53 & $*$ & & & 0.32 & $*$ & & & & & & & & & 0.96 & $*$ \\
\hline 14 & & Outer peripheral area & 0.61 & $*$ & 0.74 & $*$ & 0.69 & $*$ & 0.80 & $*$ & & & 0.59 & $*$ & & & 0.31 & $*$ & & & & & 0.71 & $*$ \\
\hline 15 & & Peak of x-direction & & & & & & & & & & & & & & & & & & & & & & \\
\hline 16 & & Power of $x$-direction & 0.45 & $*$ & 0.58 & $*$ & 0.52 & $*$ & 0.70 & $*$ & & & 0.71 & $*$ & & & & & & & & & 0.43 & $*$ \\
\hline 17 & & Peak of y-direction & & & & & & & & & & & & & & & 0.36 & $*$ & & & & & & \\
\hline 18 & & Power of y-direction & 0.51 & $*$ & 0.54 & $*$ & 0.54 & $*$ & 0.54 & $*$ & & & 0.44 & $*$ & & & & & & & & & 0.47 & $*$ \\
\hline 19 & & Peak of r-direction & & & & & & & & & & & & & & & & & & & & & & \\
\hline 20 & & Power of r-direction & 0.31 & $*$ & 0.34 & $*$ & 0.33 & $*$ & 0.37 & $*$ & & & 0.43 & $*$ & & & & & & & & & & \\
\hline 21 & & Height & 0.40 & $*$ & 0.39 & $*$ & 0.40 & $*$ & 0.56 & $*$ & & & 0.64 & $*$ & & & & & & & & & 0.33 & $*$ \\
\hline \multirow[t]{3}{*}{22} & & Body weight & 0.59 & $*$ & 0.48 & $*$ & 0.56 & $*$ & 0.48 & $*$ & & & 0.44 & $*$ & & & & & & & & & 0.44 & $*$ \\
\hline & & & & & & & & & & & & & & & & & & & & & & & & \\
\hline & & & 12 & & 13 & & 14 & & 15 & & 16 & & 17 & & 18 & & 19 & & 20 & & 21 & & 22 & \\
\hline 1 & Eyes open & $\mathrm{X}$-axis trajectory length & 0.68 & $*$ & 0.87 & $*$ & 0.58 & $*$ & & & & & & & 0.35 & $*$ & & & & & & & & \\
\hline 2 & & Y-axis trajectory length & 0.84 & $*$ & 0.80 & $*$ & 0.71 & $*$ & & & & & & & 0.43 & $*$ & & & & & 0.44 & $*$ & & \\
\hline 3 & & Total trajectory length & 0.78 & $*$ & 0.89 & $*$ & 0.67 & $*$ & & & & & & & 0.41 & $*$ & & & & & 0.33 & $*$ & & \\
\hline 4 & & Outer peripheral area & 0.50 & $*$ & 0.50 & $*$ & 0.73 & $*$ & & & 0.47 & $*$ & & & 0.61 & $*$ & & & & & 0.50 & $*$ & & \\
\hline 5 & & Peak of $\mathrm{x}$-direction & & & & & & & & & & & & & & & & & & & & & & \\
\hline 6 & & Power of $x$-direction & & & & & 0.44 & $*$ & & & 0.42 & $*$ & & & 0.46 & $*$ & & & & & 0.41 & $*$ & & \\
\hline 7 & & Peak of y-direction & & & & & & & & & & & 0.58 & $*$ & & & & & & & & & & \\
\hline 8 & & Power of y-direction & & & & & 0.45 & $*$ & & & & & & & & & -0.37 & $*$ & & & & & & \\
\hline 9 & & Peak of r-direction & & & & & & & & & & & 0.38 & $*$ & & & & & & & & & & \\
\hline 10 & & Power of r-direction & & & & & 0.49 & $*$ & & & 0.32 & $*$ & & & & & -0.31 & & & & & & & \\
\hline 11 & Eyes closed & $\mathrm{X}$-axis trajectory length & 0.71 & $*$ & 0.96 & $*$ & 0.56 & $*$ & & & & & & & & & & & & & & & & \\
\hline 12 & & Y-axis trajectory length & 1.00 & & 0.88 & $*$ & 0.61 & $*$ & & & & & & & 0.34 & $*$ & & & & & & & & \\
\hline 13 & & Total trajectory length & 0.93 & $*$ & 1.00 & & 0.61 & $*$ & & & & & & & & & & & & & & & & \\
\hline 14 & & Outer peripheral area & 0.70 & $*$ & 0.74 & $*$ & 1.00 & & & & 0.73 & $*$ & & & 0.74 & $*$ & & & 0.47 & $*$ & 0.47 & $*$ & & \\
\hline 15 & & Peak of $\mathrm{x}$-direction & & & & & & & 1.00 & & & & & & & & & & & & & & & \\
\hline 16 & & Power of $x$-direction & 0.40 & $*$ & 0.44 & $*$ & 0.76 & $*$ & & & \begin{tabular}{|l|}
1.00 \\
\end{tabular} & & & & 0.47 & $*$ & & & & & 0.49 & $*$ & & \\
\hline 17 & & Peak of y-direction & & & & & & & & & & & 1.00 & & -0.51 & $*$ & & & & & & & 0.33 & $*$ \\
\hline 18 & & Power of y-direction & 0.34 & $*$ & 0.43 & $*$ & 0.63 & $*$ & & & 0.61 & $*$ & & & 1.00 & & -0.36 & $*$ & 0.63 & $*$ & 0.51 & $*$ & & \\
\hline 19 & & Peak of r-direction & & & & & & & & & & & 0.63 & $*$ & & & 1.00 & & -0.58 & $*$ & & & & \\
\hline 20 & & Power of r-direction & & & & & 0.45 & $*$ & & & 0.51 & $*$ & & & 0.77 & $*$ & -0.45 & $*$ & 1.00 & & & & & \\
\hline 21 & & Height & & & & & 0.61 & $*$ & & & 0.68 & $*$ & & & 0.65 & $*$ & & & 0.55 & $*$ & 1.00 & & & \\
\hline 22 & & Body weight & & & 0.34 & $*$ & 0.49 & $*$ & & & 0.32 & $*$ & & & 0.50 & $*$ & & & 0.38 & $*$ & 0.57 & $*$ & 1.00 & \\
\hline
\end{tabular}

*: $\mathrm{p}<0.05$. The bottom left-half diagonal values show the results of men, and the upper right-half diagonal values show the results of women. 
In women, significant correlations $(r=0.56-0.97)$ over a moderate degree for GS variables were found among GS variables in eyes open and closed conditions, and significant correlations $(r=0.45-0.89)$ over a moderate degree were found between GS variables of both conditions. Significant correlations $(r=0.89$ and $r=0.63)$ over a moderate degree for PS variable were found between the power of $y$ - and r-directions in both vision conditions, a significantly high correlation $(r=0.90)$ was found between the peak of $y$ - and $r$-directions in the eyes open condition, and significant moderate correlation $(r=0.58)$ was found only in the peak of y-direction between eyes open and closed conditions. Significant moderate correlations $(r=0.39-0.76)$ were found between the power of $r$-direction and each GS variable in the eyes open condition and between the outer peripheral area and the power of $x$-direction $(r=0.73)$ and the power of $\mathrm{y}$-direction $(\mathrm{r}=0.74)$ in the eyes closed condition, but not in the peak of $\mathrm{x}$-, $\mathrm{r}-$, and $\mathrm{y}$-directions.

\subsection{Relationships among identifiable variables in eyes open and closed conditions}

Table 5 shows similar coefficients (intra-class correlation coefficients) among CGS variables in eyes open and closed conditions by gender. Significant intra-class correlation coefficients were found for the $\mathrm{x}$-axis trajectory length, outer peripheral area, and x-direction power in men and CGS variables except for the $\mathrm{y}$-axis trajectory length, $\mathrm{x}$ - and r-direction peak, and r-direction power in women, but the similarity coefficients were lower values under 0.70.

\section{Discussion}

The condition where eyes are closed destabilizes the standing posture compared with the eyes open condition because of restricted information feedback on the visual sense and field (Uchiyama \& Demura, 2007; Chen et al., 2012). If visual sense and field conditions change by a disturbance stimulus, the center of gravity position varies because of the strong impact of afferent information inputs and their processing (Grace Gaerlan et al., 2012). Therefore, the eyes closed condition, restricting the visual sense and field, has a significant effect on CGS.

The CGS variables are divided mainly into those regarding distance and scope of center of pressure shaking (GS variable) and frequency of COP shaking (PS variable). In this study, we hypothesized that the variation of GS and PS variables are higher when eyes are closed than when they are open (hypothesis 1) and examined the mean differences in the variables during the eyes open and closed conditions while standing. Our results showed that the GS variables were higher in eyes closed than in eyes open in both genders except for the outer peripheral area. Specifically, it was evident that the GS variables influence the restriction of the visual sense and field. Uchiyama and Demura (2007) reported similar results. The CGS variables were higher in the eyes closed condition compared with those in the eyes open condition. The $\mathrm{x}$ - and $\mathrm{y}$-axis trajectory lengths are variables that reflect left/right and before/after migratory distances, respectively. It implies that eyes closed affect left/right and before/after migratory distances.

Table 5. Similar coefficients (intra-class correlation coefficients: ICC) among CGS variables in eyes open and eyes closed conditions by gender

\begin{tabular}{|c|c|c|c|c|c|c|c|c|c|}
\hline & & \multicolumn{4}{|c|}{ Men } & \multicolumn{4}{|c|}{ Women } \\
\hline & & ICC & & F-value & & ICC & & F-value & \\
\hline 1 & $\mathrm{X}$-axis trajectory length & 0.26 & $*$ & 68.31 & $*$ & 0.44 & * & 124.16 & $*$ \\
\hline 2 & Y-axis trajectory length & 0.06 & & 98.03 & $*$ & 0.25 & & 202.99 & $*$ \\
\hline 3 & Total trajectory length & 0.15 & & 90.72 & $*$ & 0.34 & * & 210.18 & $*$ \\
\hline 4 & Outer peripheral area & 0.31 & $*$ & 29.28 & $*$ & 0.54 & * & 27.89 & $*$ \\
\hline 5 & Peak of $\mathrm{x}$-direction & 0.16 & & 3.21 & & 0.02 & & 1.08 & \\
\hline 6 & Power of x-direction & 0.69 & $*$ & 4.20 & $*$ & 0.38 & * & 1.76 & \\
\hline 7 & Peak of y-direction & 0.21 & & 0.07 & & 0.56 & * & 1.86 & \\
\hline 8 & Power of y-direction & 0.12 & & 0.01 & & 0.38 & $*$ & 0.43 & \\
\hline 9 & Peak of r-direction & 0.17 & & 3.03 & & 0.20 & & 1.72 & \\
\hline 10 & Power of r-direction & 0.18 & & 0.00 & & 0.22 & & 2.26 & \\
\hline
\end{tabular}

$*: \mathrm{p}<0.05$

The total trajectory length also showed a significant variation because both trajectory lengths increased. Uchiyama and Demura (2007) reported that the CGS increased during a standing posture owing to the eyes being closed. Our re- 
sults suggest that eyes closed affects not only left/right sway but also before/after sway in both genders, and this effect becomes higher when the eyes are closed. It is inferred that eyes closed increases a load of somatic sensation information (tension) to maintain stability because of the restricted visual information feedback by sense and field, and this effect influences the CGS. Conversely, the PS variables were higher only in the peak and power of x-direction and the power of r-direction in the eyes closed condition compared with those in the eyes open condition, but the effect size was minimal (under 0.06). Precisely, the PS variables do not affect the restriction of the visual sense and field. Our results support our first hypothesis regarding the GS variables.

In this study, we hypothesized that the GS and PS variables are higher in men than in women (hypothesis 2), and we examined the gender differences in both variable groups. The x-axis trajectory and the total trajectory lengths of the GS variables were higher in men in both eyes open and closed conditions and the y-axis trajectory length and the outer peripheral area in men only with eyes closed. The GS variables influenced by gender were identified and differentiated. Kitabayashi et al. (2004) also reported that gender differences exist in the CGS and that the GS variables were higher in men than in women. Conversely, the PS variables showed gender differences only in the power of three directions, and all the powers were higher in men compared with those in women. Our results suggest that no sex difference was found in the peak frequency but found in the power frequency. Therefore, our second hypothesis was supported for the GS variables and only the power of PS variables.

In this study, we hypothesized that the mutual relationships among GS and the PS variables in both eyes open and eyes closed are highly visible, and those between the GS and the PS variables are minimal (hypothesis 3). Our results showed that the GS variables are mutually related when the eyes are open and closed in both genders (men: $\mathrm{r}=$ 0.63-0.98, women: $\mathrm{r}=0.56-0.97$ ), but the PS variables related between the power of $\mathrm{y}$ - and $\mathrm{r}$-directions only (men: $\mathrm{r}=$ $0.76, r=0.77$, women: $r=0.89, r=0.63$ ). Previously, it was demonstrated that close relationships exist among GS variables (Kitabayashi et al., 2004; Demura et al., 2010). Close relations were seen in the CGS variables with the distance (the trajectory length per unit time) and area (the outer peripheral area, the rectangle area, etc.) because the shaking area becomes large if the shaking lengths of before/after and left/right become long. Conversely, the position variable has a different quality from other CGS variables (Demura et al., 2010). Our results suggest that regardless of whether the eyes are open or closed, the CGS variables had little relation to the peak frequency and PS variable in both sexes. Therefore, our third hypothesis is supported for only the mutual relationships in GS variables when the eyes are open and closed.

Furthermore, we hypothesized that the relationships between the identifiable GS and PS variables during eyes open and closed are low (hypothesis 4). Our results showed that the GS variables have a relationship between eyes open and closed in both genders (men: $r=0.48-0.80$, women: $r=0.45-0.89$ ), but the PS variables have a relationship between eyes open and closed only in the power of $\mathrm{x}$-direction in men $(\mathrm{r}=0.71)$, and only in the peak of $\mathrm{y}$-direction in women $(\mathrm{r}$ $=0.58$ ). Conversely, significantly low relationships exist between eyes open and closed (similarity coefficients: under 0.70 ) in the $\mathrm{x}$-axis trajectory length, the outer peripheral area, and the power of $\mathrm{x}$-direction in both men and women and in the total trajectory length, the peak of y-direction, and the power of y-direction in women. Therefore, our results supported our fourth hypothesis.

The present study examined two conditions (eyes open and closed) and the gender differences of CGS in a standing posture and the relationships among these CGS variables in healthy adults. In general, early childhood growth development and the gradual physical decline because of aging in the elderly are pertinent, as their physiques or postures differ from those of younger adults (Woollacott, 2000; Haddad et al., 2012). Thus, a follow-up study is needed to examine the same effects in infants, the middle-aged, and the elderly.

\section{Conclusions}

In conclusion, most GS and PS variables have a higher variation with eyes closed than with eyes open and in men than in women in young adults. Relationships between both CGS variables in eyes open and closed conditions exist in both genders, but the relationships between the GS and the PS spectrum variables differed.

\section{Funding}

This work was supported by the JSPS KAKENHI (Grant number JP18K11097). The funding source had no involvement with the study design, collection, analysis, and interpretation of data, writing of the report, and the decision to submit the article for publication. 


\section{References}

Chen, E. W., Fu, A. S., Chan, K. M., and Tsang, W. W. (2012). Balance control in very old adults with and without visual impairment. European Journal of Applied Physiology, 112, 1631-1636.

Degache, F., Serain, É., Roy, S., Faiss, R., and Millet, G. P. (2020). The fatigue-induced alteration in postural control is larger in hypobaric than in normobaric hypoxia. Scientific Reports, 10(1), 483.

Demura, S., Kitabayashi, T., and Noda, M. (2006). Selection of useful parameters to evaluate center-of-foot pressure movement. Perceptual \& Motor Skills, 103(3), 959-973.

Demura, S., Kitabayashi, T., and Noda, M. (2010). Power spectrum characteristics of sway position and velocity of the center of pressure during static up right posture for healthy people. Perceptual \& Motor Skills, 107(1), 89-98.

Demura, S., Kitabayashi, T., Noda, M., and Aoki, H. (2008). Age-stage differences in body sway during a static upright posture based on sway factors and relative accumulation of power frequency. Perceptual \& Motor Skills, 107(1), 89-98.

Fransson, P. A., Kristinsdottir, E. K., Hafström, A., Magnusson, M., and Johansson, R. (2004). Balance control and adaptation during vibratory perturbations in middle-aged and elderly humans. European Journal of Applied Physiology, 91(5-6), 595-603.

Grace Gaerlan, M., Alpert, P. T., Cross, C., Louis, M., and Kowalski, S. (2012). Postural balance in young adults: the role of visual, vestibular and somatosen sory systems. Journal of the American Association of Nurse Practitioners, 24, 375-381.

Haddad, J. M., Claxton, L. J., Keen, R., Berthier, N. E., Riccio, G. E., Hamill, J., and Van Emmerik, R. E. (2012). Development of the coordination between posture and manual control. Journal of Experimental Child Psychology, 111(2), 286-298.

Hill, H. W., and Price, M. J. (2018). Carrying heavy asymmetrical loads increases postural sway during quiet standing in older adults. Aging Clinical and Experimental Research, 30(9), 1143-1146.

Kapteyn, T. S., Bles, W., Njiokiktjien, C. J., Kodde, L., Massen, C. H., and Mol, J. M. (1983). Standardization in platform stabilometry being a part of posturography. Agressologie, 24, 321-326.

Kitabayashi, T., Demura, S., Noda, M., and Yamada, T. (2004). Gender differences in body-sway factors of center of foot pressure in a static upright posture and under the influence of alcohol intake. Journal of Physiological Anthropology Applied Human Sciences, 23(4), 111-118.

Masani, K., Vette, A. H., Kouzaki, M., Kanehisa, H., Fukunaga, T., and Popovic, M. R. (2007). Larger center of pressure minus center of gravity in the elderly induces larger body acceleration during quiet standing. Neuroscience Letters, 422(3), 202-206.

Noda, M., Demura, S., Yamaji, S., and Kitabayashi, T. (2004). Influence of alcohol intake on the parameters evaluating the body center of foot pressure in a static upright posture. Perceptual \& Motor Skills, 98(3 Pt 1), 873-887.

Noguchi, T., Demura, S., and Nakagawa, T. (2013). Postural stability during one-leg stance on an unstable moving platform and its relationship with each leg. Perceptual \& Motor Skills, 116(2), 555-563.

Pascoe, D. D., Pascoe, D. E., Wang, Y. T., Shim, D. M., and Kim, C. K. (1997). Influence of carrying book bags on gait cycle and posture of youths. Ergonomics, 40(6), 631-641.

Siriphorn, A., Chamonchant, D., and Boonyong, S. (2015). The effects of vision on sit-to-stand movement. Journal of Physical Therapy Science, 27(1), 83-86. doi: 10.1589/jpts.27.83. Epub 2015 Jan 9.

Society for Physical Fitness Standards Research in Tokyo Metropolitan University. (2000). [New Physical Fitness Standards of Japanese People] (pp. 20-85). Tokyo: Fumaido. [in Japanese]

Taguchi, K., Kikukawa, M., Miyashita, Y., Goto, A., Ito, K., and Fukazawa, O. (1986). Relationship between movements of the head and the center of gravity of the body in Menière's disease. Auris Nasus Larynx, 13 (Suppl 2), S161-169.

Uchiyama, M., and Demura, S. (2007). Low visual acuity is associated with the decrease in postural sway. The Tohoku Journal of Experimental Medicine, 212(1), 13-20. doi: 10.1620/tjem.212.13.

Vuillerme, N., and Nougier, V. (2003). Effect of light finger touch on postural sway after lower-limb muscular fatigue. Archives of Physical Medicine and Rehabilitation, 84(10), 1560-1563.

Woollacott, M. (2000). Systems contributing to balance disorders in older adults. The Journals of Gerontology: Series A, 55(8), M424-M428.

Yoneda, S., and Tokumasu, K. (1986). Frequency analysis of body sway in the upright posture: Statistical study in cases of peripheral vestibular disease. Acta Oto-Laryngologica, 102(1-2), 87-92. doi: 10.3109/00016488609108650. 